\title{
Cancerogene Wirkung von Kunststoff-Folien ${ }^{\dagger}$
}

\author{
Von Hermann Druckrey und Dietrich Schmähl \\ Aus der chirurgischen Universitätsklinik in Freiburg i. Br. (Direktor: Prof. Dr. E. Rehn) \\ (Z. Naturforschg. 7 b, 353-356 [1952]; eingegangen am 3. April 1952)
}

1. In Übereinstimmung mit $\mathrm{O} p \mathrm{p}$ e $\mathrm{n}$ h e i m e r wird festgestellt, daß Folien aus regenerierter Cellulose bei Implantation an Ratten cancerogen sind.

2. Zerstoßene Glaswolle erzeugte in Kontrollversuchen keinen Krebs.

3. Mit siedendem Benzol extrahierte und reine, ohne Zusatz hergestellte Folien aus regenericrter Cellulose waren ebenso cancerogen wie das Handelsprodukt.

4. Die beobachtete cancerogene Wirkung der Folien wird mit der "Silikose“ erzeugenden Wirkung polymerer Silikate und der Krebs erzeugenden Wirkung von Asbest, von bifunktionellen Methylolamiden, Äthyleniminen, Epoxyden sowie von $N$-Lost-Verbindungen verglichen und darauf bezogen, daß sich funktionswichtige Zellproteine mit den linearen Makromolekülen durch Restvalenzen (Wasserstoff-Bindungen) vernetzen und damit verändert werden.

5. Die hiernach zu erwartende cancerogene Wirksamkeit von Polyamid-Folien wird an Ratten festgestellt.

$\mathrm{N}$ ach Implantation von Plättchen aus dem Kunststoff „Bakelit“ unter die Haut von Ratten beobachtete T urner ${ }^{1}$ die lokale Entstehung von Sarkomen. Aus diesem Grunde haben wir untersucht, ob auch andere Kunststoffe eine krebserzeugende Wirkung haben. Über die Ergebnisse der 1949 begonnenen Versuche, die an insgesamt 281 Ratten durchgeführt wurden, soll hier kurz berichtet werden.

Ratten unserer Inzuchtstämme BD I und BD III, bei denen seit 1937 niemals spontan Sarkome aufgetreten sind, erhielten mehrere pfenniggroße Scheibchen handelsüblicher Folie aus regenerierter Cellulose (Hydrocellulose) unter die Haut bzw. in die Bauchhöhle eingepflanzt. Insgesamt 13 von den länger als 1 Jahr überlebenden 50 Ratten entwickelten lokal Sarkome** (Abb. 1-3*).

Den gleichen Befund hatte vor uns Oppenhei $\mathrm{me} \mathrm{r}^{2}$ erhoben, der ebenfalls nach Implantation von Cellulose-Folien an Ratten Sarkome beobachtete. Seine Ergebnisse, die uns aus äußeren Gründen erst vor 2 Jahren bekannt wurden, stimmen mit den unseren in allen Punkten überein.

Die damit gesicherte krebserzeugende Wirkung der Cellulose-Folie kann nicht auf Verunreinigungen oder Zusätze bezogen werden, denn wir fanden Extrakte, die aus den Folien mit siedendem Benzol hergestellt waren, nicht cancerogen wirksam. Dagegen erzeug-

* Abb. $1-3$, s. Tafel S. 353 a.

** Für die histologische Diagnose danken wir den Herren Prof. H. H a m p e r l, Marburg, und Dr. W. A l t m a n n, Freiburg.

1 F. C. T u rner, J. nat. Cancer Inst. 2, 81 [1941]. ten die vorher mit Benzol extrahierten Folien ebenso Krebs (Abb.2), wie das Ausgangsmaterial und wie Folien, die für diesen Zweck aus regenerierter Cellulose ohne jeden Zusatz hergestellt waren (Abb. 3). Die krebserzeugende Wirkung kommt also der polymeren Hydrocellulose selbst zu.

Die Geschwülste traten zwischen dem 190. und 600. Tag nach der Implantation auf. War diese unter die Haut erfolgt, so entstand meist ein einzelner größerer Krebsknoten, in dessen nekrotischer Höhle die Plättchen lagen (Abb. 1). Die Ratten, denen die Plättchen in die Bauchhöhle eingepflanzt waren, zeigten dagegen meist zahlreiche kleinere Sarkome, die von allen Stellen ausgingen, an denen die Plättchen einmal gelegen haben mochten (Abb. 2 und 3). Diese selbst lagen meist bei der Sektion frei in der Bauchhöhle und erschienen auch nach mehr als 15 Monaten unverändert. Die cancerogene Wirksamkeit der Folien muß danach stark sein. Zur Auslösung von Krebs scheint eine Zeitdauer des direkten Kontaktes zu genügen, die wesentlich kleiner ist als die Latenzzeit bis zum Auftreten der Geschwülste.

In Fütterungsversuchen mit zerkleinerter Folie beobachteten wir bisher nach 2 Jahren einen Fall von lymphatischer Leukämie (Lymphosarkom) mit bösartigen Infiltraten in den Lymphknoten, der Leber, der Milz und der Lunge.

+ Die Arbeiten wurden durch die D e u t s che F or s chungs gem e in s ch a f t ermöglicht.

2 B. S. O p pe n h e i m er, T. E. O p p e n he i m e r u. A. P. S t o u t, Transact. Ass. Amer. Physicians 1948, 343; Proc. Soc. exp. Biol. Med. 67, 33 [1948]. 
Kontrollversuche, in denen wir 12 Ratten zerstoBene Glaswolle in die Bauchhöhle implantiert hatten, lieferten keine Geschwulst. Entsprechend der allgemeinen experimentellen Erfahrung ist der chronische mechanische Reiz allein also nicht cancerogen. Die cancerogene Wirkung der Folien muß daher auf die Substanz der Folie bezogen werden. -

Die linearen, makromolekularen Hauptvalenzketten der Cellulose sind in den Folien durch Nebenvalenzkräfte zu einem mizellaren Verband verknüpft, dessen Ordnungszustand wechselt und nicht definiert ist. In Wasser quellen die Folien, indessen ist eine Lösung in den Körperflüssigkeiten kaum denkbar. An den Oberflächen der Folien liegen dagegen Bindungskräfte frei, durch die z. B. Farbstoffe sehr fest gebunden werden können. Die Tatsache, daß fluoreszierende Substanzen von den Folien nicht abgewaschen werden können, wird sogar als Unterscheidungsmerkmal für Hydrocellulose-Folien von Folien anderer Art benutzt ${ }^{3}$.

Danach liegt der Gedanke nahe, daß auch lineare Zellproteine, die in geeigneter Orientierung mit der gequollenen Folienoberfläche in genügend engen Kontakt kommen, von den ebenfalls linearen Makromolekülen der Kunststoffe z. B. durch WasserstoffBindungen sehr fest gebunden werden können, und daß auf diese Weise die krebsige Entartung von Zellen bewirkt wird. Auch eine Ablösung einzelner, so mit einem Zellprotein verbundener Kunststoff-Moleküle aus der Oberfläche erscheint denkbar. Damit würde gleichzeitig eine Regeneration der aktiven Oberfläche verbunden sein.

Für einen solchen Mechanismus sprechen zahlreiche Beobachtungen aus der letzten Zeit. E a r l $\mathrm{e}^{4}$ fand an Gewebekulturen, die auf Hydrocellulose-Folien gezüchtet waren, in vitro eine „spontane“ Cancerisierung normaler Zellen, die durch Retransplantation der Kulturen auf gleichartige Tiere bewiesen wurde. Nach den Befunden von Op pen he i mer ${ }^{2}$ und uns halten wir es für möglich, daß die beobachtete Cancerisierung auch hier den Kunststoff-Folien zuzuschreiben ist.

3 G. S a n d ó r, Z. angew. Chem. 42, 1108 [1937].

${ }^{4}$ K. K. S a nford, W. R. Earle, E. Shelt on, E. L. S chilling, E. M. Duchesne, G. D. Likely u. M. M. B e c k e r, J. nat. Cancer Inst. 11, 351 [1950].

5 R. J ä g e r, Kolloid-Z. 119, 165 [1950]. Vortrag Silikose-Tagung Münster 19. Oktober 1949. „Kolloidchemische Gesichtspunkte in der Staublungenforschung “ in JöttenGärtner : „Die Staublungenerkrankungen “. Darmstadt 1950. - „Staublungenforschung und Gewerbehygiene“, zusammengestellt von K. W. J ö t t e n, Bundesarbeitsblatt 1951, Nr. 10.
Ein ähnlicher Wirkungsmechanismus ist tür die „Silikose“ erzeugenden Silikate anzunehmen ${ }^{5}$. Monomere und amorphe polymere Silikate erzeugen keine Silikose, dagegen kristalline Polymere, und zwar um so schneiler und stärker, je höher der Ordnungszustand in den Silikaten ist ${ }^{5}$. Das Ladungsmuster der Kristallite stimmt mit dem im Kollagen vorhandenen auffallend überein. Auch hier wird es als wahrscheinlich angenommen, daß Zellproteine von den linearen polymeren Silikatmolekülen an der Oberfläche gebunden werden und daß sich die gebildeten Aggregate abïösen können, wobei die aktive Oberfläche wieder regeneriert wird ${ }^{6}$. Inzwischen wurde die relativ gute Löslichkeit der oberflächlichen Lagen von Quarzpartikelchen nachgewiesen ${ }^{6 a}$. Für eine Lösung spricht schon der Nachweis von Kieselsäure in den Körperflüssigkeiten. Die Quarzteilchen können von Zelle zu Zelle weitergereicht werden und diese dabei verändern, ohne ihre Aktivität zu verlieren (W. G i e s e, zit. n. J ä g e r ${ }^{5}$ ). Die „Silikose“ erzeugende Wirkung stimmt pharmakologisch insofern mit der krebserzeugenden Wirkung überein, als beide irreversibel sind und beim Menschen oft erst Jahrzehnte nach der Exposition manifest werden und zum Tode führen können.

Im Asbest, der ebenfalls im Körper nicht löslich ist und trotzdem sowohl beim Menschen ${ }^{7}$ als auch experimentell am Tier $^{8}$ Lungenkrebs erzeugt, liegt ein weiteres Beispiel für die cancerogene Wirksamkeit polymerer Substanzen aus linearen Makromolekülen vor.

Der gleiche Wirkungsmechanismus ist nach neuesten Untersuchungen auch für vier andere Stoffklassen wahrscheinlich geworden, die eine ,radiomimetische" Wirkung auf Zellen haben und sowohl cytotoxisch als auch mutagen und cancerogen sind, nämlich Methylolamide, Äthylenimime, Epoxyde und die in vivo zu Äthyleniminen bzw. zu Epoxyden veränderbaren Stickstoff-Lost-Verbindungen (Tafel 1).

Einige derartige Substanzen wurden in Deutschland seit längerer Zeit zur Veredelung von Textilien verwendet. Sie erzeugen bleibende Veränderungen an Cellulose- oder Proteinfasern, und zwar dadurch, daß

6 R. J äg e r, Wechselwirkungen zwischen Quarz und Organismus. Vortrag Bochum 1951 im Druck in: Beiträge Silikoseforschg., Heft 15. Bochum 1952.

6a E. J. K ing, Occup. Med. 4, 26 [1947]; P. B. D e m p s t e r u. P. D. Ri t c h i e, Nature [London] 169, 538 [1952].

7 H. W. W e d l e r, Dtsch. med. Wschr. 69, 575 [1943].

8 M. N ordmann u. A. S or g e, Z. Krebsforschg. 51, 168 [1941]; W. C. H u e per, „Occupational and environmental cancer“, Publ. Health Rep. 1948, 209. 
Methylolamide:

\section{$\mathrm{HOH}_{2} \mathrm{C} \cdot \mathrm{NH} \cdot \mathrm{R} \cdot \mathrm{NH} \cdot \mathrm{CH}_{2} \mathrm{OH}$}

Äthylenimine:<smiles>C1CN1</smiles>

Epoxyde:<smiles>[R]C1CO1</smiles>

$N$-Lost-Verbindungen:<smiles>[R]N(CCCl)CCCl</smiles><smiles>[R][N+]1(CCCl)CC1</smiles><smiles>[R]N1CCOC1CCl</smiles>

Tafel 1. Cancerogene Substanzen (nach H e n d r y ${ }^{11}$ ).

sie durch Restvalenzen längstseits von den Fasern gebunden werden oder Vernetzungen herstellen. Mit diesen Substanzen wurden in vitro auch Veränderungen an Nucleinsäuren beobachtet ${ }^{9}$. Bei Einwirkung auf sich teilende Zellen erzeugen sie sichtbare Abnormalitäten an Chromosomen ${ }^{10}$, die denen nach Strahlenwirkung entsprechen (,radiomimetische Wirkung").

Eine cancerogene Wirkung kommt vornehmlich den bifunktionellen Molekülen zu, also den bis-Methylol-amiden, bis-Epoxyden, bis-Äthyleniminen und bis-[Chloräthyl]-alkyl-aminen ${ }^{11}$. Diese Substanzen geben in vitro wahrscheinlich Radikale, jedenfalls bilden sie mit Leichtigkeit lineare Polymere.

Die cytotoxische, mutagene und auffallend starke cancerogene Wirkung von Substanzen dieser Art wird darauf bezogen, daß die Monomeren im Körper lineare Polymere bilden, die sich dann ähnlich, wie das in vitro mit Cellulose, Proteinen und Nucleinsäuren beobachtet wurde ${ }^{9}$, mit funktionswichtigen Proteinen oder Nucleoproteinen der Zelle, die „Du-

9 D. T. Elmore, J. M. Gull and, D. O. J or d a n u. H. F. W. T a y lo r, Biochem. J. 42, 308 [1948].

10 R. J. Gold a cre, A. Loveles u. W. C. J. R o s s, Nature [London] 163, 667 [1949].

11 F. S. Phillips, J. Pharmacol. exp. Therapeut. 99, 281 [1950]; J. A. H e n d r y, F. L. R o s e u. A. L. Wa lp o l e, Brit. J. Pharmacol. Chemotherapy 6, 201 [1951]; J. A. H endry, F. L. Ros e, A. L. W a l pole u. R. F. H o m e r, Brit. J. Pharmacol. Chemotherapy 6, 235, 357 [1951].
plikanten“-Eigenschaft besitzen, vernetzen oder der Länge nach verknüpfen und sie so irreversibel verändern oder blockieren ${ }^{11}$. Daß im Körper derartige Polymerisationen tatsächlich stattfinden können, und zwar auch solche, die in vitro nur mit Katalysatoren verlaufen, wurde kürzlich am Beispiel des Acrylamids gezeigt ${ }^{12}$, also einer Grundsubstanz für wichtige Kunststoffe. Auf cancerogene Wirkung wurde Acrylamid noch nicht untersucht. Die einfachste cancerogene Substanz aus diesen Reihen ist das $\mathrm{Bu}-$ tadien-diepoxyd $^{13}(\mathrm{I})$.<smiles>C1OC1C1CO1</smiles>

Die Polymeren der aktivsten Substanzen dieser Reihen zeigen zwischen den brückenbindungsfähigen Atomen Abstände von 3,7 bzw. 7,5 $\AA$, die denen zwischen den Purin- bzw. Pyrimidin-Kernen in Nucleinsäuren entsprechen ${ }^{14}$. Das gilt aber nicht streng, vielmehr sind auch Substanzen mit abweichenden Abstandswerten noch wirksam. Dagegen sind Substanzen, deren Polymere keine BrückenbindungsFähigkeiten haben, nicht aktiv. Damit ist es wahrscheinlich, daß nicht nur das Vorhandensein von freien Restvalenzen, genauer: von $\pi$-Elektronen- oder Proton-Donatoren für die genannten pharmakologischen Wirkungen wesentlich ist, sondern auch ihre räumliche Ordnung, z. B. ihr linearer Abstand voneinander in den Polymeren.

Hendry und Mitarbb. diskutieren einen solchen Wirkungsmechanismus auch für die cancerogenen aromatischen Kohlenwasserstoffe und Amine ${ }^{11}$. An anderer Stelle ${ }^{15}$ haben wir gezeigt, daß die Eigenschaften von aromatischen Aminen, die ihre cancerogene Wirksamkeit bestimmen, in vielen Punkten mit den Eigenschaften von Farbstoffen übereinstimmen, die ihre "Substantivität“ bedingen, d. h. ihre Fähigkeit, auf lineare Cellulosefasern „direkt“ aufzuziehen ${ }^{16}$. Solche Gesichtspunkte erscheinen für die Pharmakologie deshalb bedeutsam, weil die wesentlichen Funktionsträger in der Zelle, vor allem solche

12 G. W il ke, Naturwiss. 38, 532 [1951].

13 J. A. Hendry u. Mitarbb., l. c. 11.

14 J. K. Dixon, G. L. M. Christopher u. D. L. S a ll e y, Paper Trade J. 127, 455 [1948]; J. A. H e n d ry u. Mitarbb., l. c. 11 .

15 H. Druckrey, D. Schmähl u. P. Danneb e r g, Naturwiss. 39 [1952], i. Druck.

16 Th. Vikkerstaff, "The physical chemistry of dyeing" Oliver \& Boyd, London 1950. 
mit „Duplikanten“-Eigenschaft, lineare Makromoleküle sein dürften ${ }^{17}$.

Als Voraussetzung für die Bindung an lineare Makromoleküle der Zelle muß das Vorhandensein von Restvalenzkräften in den Kunststoffen angenommen werden. Wir haben deshalb eine Polyamidfolie, die hiernach aktiv sein müßte, auf cancerogene Wirkung untersucht, und zwar $\varepsilon$-Caprolactam-Folien (II).<smiles>CCCNC(=O)CCCCCNC(=O)CCC</smiles>

Struktur der $\varepsilon$-Caprolactam-Polymere

9 Ratten wurden je 5 Folienstückchen von Pfenniggröße in die Bauchhöhle implantiert. Nach 360 Tagen lebten noch 6 Tiere. Vier davon haben bisher multiple und sehr bösartige Sarkome entwickelt, die über den ganzen Bauchraum verteilt waren (Abb. $\left.4^{*}\right)$. Die Wirkung entspricht vollkommen der von Cellulosefolien und scheint sogar stärker zu sein als diese.

Hendry ${ }^{11}$ berichtet in einem Nachtrag seiner letzten Arbeit, daß das dem $\varepsilon$-Caprolactam Polymerisat sehr nahe verwandte Caproyl-äthylen-imin (III) stark cancerogen sei. Orientierende Versuche<smiles>CCCCCC(=O)N1CC1</smiles>

\section{Caproyl-äthylenimin}

mit einigen Kunsttextilien, wie Orlon, Nylon- und Perlongewebe verliefen bisher negativ, obwohl die Beobachtungszeit mehr als 16 Monate beträgt. Da

17 H. S t a u d i n g e r, "Makromolekulare Chemie und Biologie“, Wepf, Basel 1947.

18 Doerr, B leyert u. S chmidt, Z. Krebsforschg. 36, 256 [1932]. diese Gewebe sich nur gestaltlich, kaum aber chemisch von den entsprechenden Folien unterscheiden, muß es für möglich gehalten werden, daß auch die mechanische Verletzung von Zellen durch die scharfe Kante der Folien bei ihrer cancerogenen Wirkung eine Rolle spielt und vielleicht diese einleitet. Fär sich allein kann der chronische mechanische Reiz indessen keinen Krebs erzeugen, wie der negative Ausgang unserer Kontrollversuche, in denen zerstoBene Glaswolle implantiert wurde, erneut zeigt. Die substantielle Wirkung der Folien muß daher das Wesentliche sein. So mag der Mechanismus dieser cancerogenen Wirkung von linearen Polymeren ähnlich liegen wie der bei der Infektion mit dem Rou sSarkom-Virus, die ebenfalls eine Verletzung von Zellen voraussetzt ${ }^{18}$. Die aufgeworfenen Probleme sind dem Experiment zugänglich.

Nach den bisher vorliegenden Befunden muß gefordert werden, daß die Verwendung polymerer Kunststoffe als plastische Massen in der Chirurgie ihre gründliche Prüfung in Dauerversuchen am Tier voraussetzt. Die cancerogen gefundenen Kunststoffe dürfen für diesen Zweck nicht verwendet werden.

$\mathrm{N}$ a c htrag b. d. K orr.: Kürzlich teilten O p pen h e i m e r u. Mitarbb. ${ }^{19}$ weitere Befunde mit Kunststoffen an Ratten und Mäusen mit. Genuine Cellophanfilme erzeugten in etwa $36 \%$ der Fälle Sarkome, vorher mit Alkohol extrahierte Filme sogar in 45\%. Der Alkoholextrakt war dagegen wirkungslos. Kontrollversuche, in denen Glasplättchen oder Watte implantiert waren, ergaben keinen Krebs. Diese Ergebnisse decken sich völlig mit den unsern. Vinylchloridfilme waren etwa ebenso wirksam wie Cellophanfilme, Polyäthylenfolien dagegen deutlich schwächer $(13 \%)$, wie es nach den hier entwickelten Vorstellungen über die Bedeutung brückenbindungsfähiger Gruppen für die Wirksamkeit erwartet werden mußte.

* Abb. 4, s. Tafel S. 353 a.

19 Briefl. Mitteilung und B. S. O p p e $\mathrm{n}$ h e i m e r, E. T. O p p e n h e i m e r u. A. P. S t o u t, Proc. Soc. exp. Biol. Med. 79, 366 [1952]. 